\title{
POSTER
}

\section{Kératokyste odontogène orthokératinisant et tumeur odontogène kératokystique : intêret de l'immunohistochimie}

\section{Castro $\mathrm{R}^{1}$, Ordioni $\mathrm{U}^{1,2}$, Del Grande $\mathrm{J}^{3}$, Laurent $\mathrm{P}^{1}$, Hoaraux $\mathrm{MO}^{1}$, Catherine $\mathrm{JH}^{1,4}$}

1 - Service d'Odontologie, Assistance Publique Hôpitaux de Marseille, Hôpital de la Timone, 264 rue Saint Pierre, 13005 Marseille

2 - Centre Massilien de la Face, 24 avenue du Prado, 13006 Marseille

3 - Service d'Anatomie Pathologique et de Cytologie, Assistance Publique Hôpitaux de Marseille, Hôpital de la Timone, 264 rue Saint Pierre, 13005 Marseille, France

4- UMR 7268 ADES, Aix-Marseille Université/EFS/CNRS, Faculté de Médecine-secteur Nord, Bd Pierre Dramard, 13344, Marseille Cedex 15

\section{Introduction}

Les kératokystes odontogéniques ont été décrits en 1956 par Philipsen et al. Ils se caractérisent par la présence d'un épithélium endocavitaire pavimenteux, différencié et kératinisant. En 2005, I'OMS différencie les kératokystes en fonction du type de kératinisation. Le type orthokératinisé est appelé kératokyste odontogénique orthokératinisant (KOO). La variante parakératinisée correspond à la tumeur odontogène kératokystique (TOK). Cette forme parakératinisée est considérée comme une tumeur bénigne mais possédant un potentiel agressif local et récidivant. Le diagnostic différentiel histologique peut être difficile. II peut en effet exister des remaniements inflammatoires qui tendent à faire disparaître la kératinisation. L'aspect de la couche basale et l'immunohistochimie pourraient apporter des éléments supplémentaires pour le diagnostic de ces lésions.

\section{Observations}

Trois cas de kératokystes illustrent cette difficulté diagnostique. À partir de la littérature, la place de l'immunohistochimie est discutée dans le diagnostic et l'évaluation du caractère récidivant de la lésion.

\section{Discussion}

Certains marqueurs biologiques ont été étudiés afin de caractériser ces lésions et de comprendre la différence de comportements cliniques et biologiques entre les formes ortho et parakératinisées. Ainsi, il existerait une augmentation significative de l'expression de la P53 dans les couches suprabasales dans les TOK par rapport aux KOO et aux autres kystes odontogènes (Deyhimi et al, 2014). Une mutation de la P53 est observée dans le TOK (Gadbail, 2012). La protéine P63 est exprimée intensément dans les couches supra-basales du TOK. Elle l'est moins dans le KOO et les autres kystes odontogènes.

L'antigène Ki-67 est considéré comme un marqueur spécifique de la prolifération cellulaire, son expression serait augmentée au niveau de la couche suprabasale dans les TOK. Cela expliquerait son caractère envahissant et recidivant. De plus, l'expression de l'antigène PCNA est plus intense dans les TOK au niveau des cellules supra-basales. Ces observations seraient en faveur 
de l'existence d'une anomalie dans le contrôle du cycle cellulaire dans la TOK et expliquerait son comportement agressif.

Il existe également une augmentation du TGF-Alpha dans les TOK qui pourrait expliquer le taux de transformation maligne plus important dans ce type de lésions (Deyhimi et al, 2014).

Au niveau du diagnostic différentiel, la forme orthokératinisée du kératokyste se distinguerait de la forme parakératinisée par l'absence d'expression de la CD-56, de la Calrétinine, de la GP-38 et la basse expression de la P63, de l'IPO-38, de la CD-105 et de la CD-10 (Vera-Sirera et al, 2015).

\section{Conclusion}

L'expression de facteurs biologiques au sein d'une lésion et leur utilisation en tant que marqueurs cellulaires est une donnée nouvelle et significative qui doit être prise en considération dans l'établissement d'un diagnostic positif et le suivi des patients.

\section{Références}

Deyhimi et al. Pathol Res Pract. 2014 Apr;210(4):201-4.

Gadbail et al. Acta Odontol Scand. 2012 Dec;70(6):529-35.

Vera-Sirera et al. Head Face Med. 2015 Feb 12;11:3. 